\title{
CAPNet: Continuous Approximation Projection for 3D Point Cloud Reconstruction Using 2D Supervision
}

\author{
K. L. Navaneet," Priyanka Mandikal," Mayank Agarwal, R. Venkatesh Babu \\ Video Analytics Lab, CDS, Indian Institute of Science, Bangalore, India \\ navaneetl@iisc.ac.in, priyanka.mandikal@gmail.com, mayankgrw197@gmail.com, venky@iisc.ac.in
}

\begin{abstract}
Knowledge of 3D properties of objects is a necessity in order to build effective computer vision systems. However, lack of large scale 3D datasets can be a major constraint for datadriven approaches in learning such properties. We consider the task of single image 3D point cloud reconstruction, and aim to utilize multiple foreground masks as our supervisory data to alleviate the need for large scale 3D datasets. A novel differentiable projection module, called 'CAPNet', is introduced to obtain such $2 \mathrm{D}$ masks from a predicted $3 \mathrm{D}$ point cloud. The key idea is to model the projections as a continuous approximation of the points in the point cloud. To overcome the challenges of sparse projection maps, we propose a loss formulation termed 'affinity loss' to generate outlierfree reconstructions. We significantly outperform the existing projection based approaches on a large-scale synthetic dataset. We show the utility and generalizability of such a 2D supervised approach through experiments on a real-world dataset, where lack of $3 \mathrm{D}$ data can be a serious concern. To further enhance the reconstructions, we also propose a test stage optimization procedure to obtain reconstructions that display high correspondence with the observed input image.
\end{abstract}

\section{Introduction}

3D Reconstruction from images is a key challenge in the field of computer vision. While deep learning based approaches have achieved exceptional results in various computer vision tasks (Krizhevsky, Sutskever, and Hinton 2012; Girshick et al. 2014; Long, Shelhamer, and Darrell 2015; Goodfellow et al. 2014), the capability of such approaches is limited by the amount of data available. Obtaining large scale 3D data of objects can be expensive and timeconsuming. In contrast, capturing 2D data (image, foreground mask etc.) from multiple view points is relatively easy. We consider the task of single image 3D point cloud reconstruction and aim to utilize such $2 \mathrm{D}$ observations in place of point clouds as our supervisory data. Towards this end, we propose a novel differentiable projection module to obtain the 2D observations from the predicted points.

The nature of the projection module is dependent on our choice of the 3D representation. Unlike 2D images, where

\footnotetext{
*equal contribution Copyright (C) 2019, Association for the Advancement of Artificial Intelligence (www.aaai.org). All rights reserved.
}

all the pixels add rich spatial and structural information, volumetric representations suffer from sparsity of information. The information needed to perceive the 3D structure is provided by surface voxels, while the voxels within the volume increase the representational and computational complexity with minimal addition in information. 3D point clouds are a more efficient alternative, since the points are sampled on the surface of the object. However, lack of grid structure and permutation invariance properties of point clouds pose challenges in their processing. Recent works address these issues using point cloud specific architectures and loss formulations (Qi et al. 2017a; 2017b; Fan, Su, and Guibas 2017; $\mathrm{Su}$ et al. 2018; Li et al. 2018).

In the case of 3D voxel-based approaches, the projection is obtained via a transformation between two grid representations and hence can be performed by simple interpolation operations (Yan et al. 2016). Point clouds, however, pose two important challenges: (1) Firstly, projecting low density point clouds using conventional interpolation techniques can result in projections with holes. Generating high density point clouds requires higher memory and computational power. (2) Secondly, the process of obtaining the projections by discretizing the point cloud is a non-differentiable operation. To address both these issues, we propose a continuous approximation of points in the point cloud which produces smooth projections in a differentiable manner.

However, a sparse projection map results in very low gradients in regions where no point is projected, which leads to outlier points. We propose a novel loss function, termed Affinity Loss, to enforce effective flow of gradients in such situations, resulting in cleaner and better reconstructions.

Since 2D observations like foreground masks can be obtained from the input image, projection based approaches provide a unique opportunity for optimization on the test data. Given a test image, the point cloud obtained using a pre-trained networked can be modified to exactly match the corresponding mask. We introduce such a set-up and obtain more accurate reconstructions with improved correspondence to the input image.

To summarize, we make the following key contributions:

- We propose CAPNet, a continuous approximation projection module for a differentiable and accurate rendering of $3 \mathrm{D}$ point clouds, to enable weakly supervised 3D object reconstruction. The proposed rendering module generates 
smooth, artifact-free projections, while also overcoming the lack of gradients that can exist in a naive discretization based approach.

- We formulate a loss function termed Affinity Loss for effectively penalizing outlier points, resulting in reconstructions of high quality.

- Using as little as a single mask as supervision, we finetune a 3D supervised network on a real world dataset, and demonstrate the efficacy of the approach in obtaining superior reconstructions.

- We perform extensive quantitative and qualitative evaluation of CAPNet on synthetic and real datasets, and show that it significantly outperforms the state-of-the-art projection-based reconstruction methods.

- Finally, we present a technique for optimizing the predicted point clouds at test time using available foreground masks, and obtain reconstructions that highly correspond to the input image.

\section{D Supervision}

\section{Related works}

A number of 3D reconstruction works employ training procedures that utilize the complete 3D data available. With the advent of deep neural network architectures in 2D image generation tasks, the power of convolutional neural nets have been directly transferred to the 3D domain using 3D CNNs. There is vast literature on generating voxelized output representations. (Girdhar et al. 2016) learnt a joint embedding of 3D voxel shapes and their corresponding 2D images. (Choy et al. 2016) trained a recurrent neural network to encode information from many input views. These works predict voxelized outputs and utilize 3D voxel-based losses for supervision. But the compute overhead and sparsity of information in voxel formats inspired lines of work that abstracted volumetric information into smaller number of units with the help of the octree data structure (Tatarchenko, Dosovitskiy, and Brox 2017; Riegler, Ulusoy, and Geiger 2017; Häne, Tulsiani, and Malik 2017). More recently, Fan et al. (Fan, Su, and Guibas 2017), introduced frameworks and loss formulations tailored for generating unordered point clouds, and achieved single-view 3D reconstruction results outperforming the volumetric state-of-art approaches (Choy et al. 2016). Several other recent works tackle the problem of 3D point cloud reconstruction from a single image (Groueix et al. 2018; Mandikal et al. 2018; Mandikal, K L, and Babu 2018; Mandikal and Babu 2019). While all of the above works directly use full 3D point cloud supervision, we show competitive $3 \mathrm{D}$ reconstruction capability by using only $2 \mathrm{D}$ masks as supervision.

\section{D Supervision}

Recent works have explored ways to reconstruct 3D shapes from 2D projections such as silhouettes and depth maps with the help of differentiable rendering modules.

(1) Volumetric Rendering: Perspective transformer nets (PTN) (Yan et al. 2016) performs perspective transformation and grid sampling of volumetric outputs to obtain the projections. Tulsiani et al. (Tulsiani et al. 2017) use differentiable ray consistency loss to train on 2D observations like foreground mask, depth, color images and semantic maps. MarrNet (Wu et al. 2017) predicts normal, depth and silhouette maps from 2D images and reconstructs voxel outputs using the estimated 2.5D sketches, while also enforcing projection consistency at test time.

(2) Point-cloud Rendering: Amongst the point-cloud based works, Lin et al. (Lin, Kong, and Lucey 2018) use a combination of depth fusion and point projection as supervision. Our approach differs from Lin et al. in the following ways: (a) In Lin et al., the network is first pretrained without using projection loss, but directly regressing for depth maps from eight fixed views, which are fused to obtain the point cloud. The projection loss is only used for fine-tuning this model once it has been trained. On the other hand, our model requires no pretraining and as little as a single mask from a random view can be used as supervision. (b) The projection module in Lin et al. consists of discretizing the $(x, y)$ coordinates of the point cloud and projecting the $z$ values onto a plane. To avoid point collisions, a memory intensive operation (termed 'upsampling') is performed. In contrast, we directly obtain the projected map via the continuous approximation module. Further, we introduce the Affinity Loss in the training regime to remove outlier points.

Apart from volumetric and point-cloud based approaches, differentiable rendering modules for 3D meshes have also been proposed (Kato, Ushiku, and Harada 2017).

\section{Problem Formulation}

\section{Approach}

Given a single image of an object, we aim to reconstruct its $3 \mathrm{D}$ point cloud representation. Let $I$ be an image from the training set. Let $p=f(I)$ be the corresponding 3D point cloud reconstruction obtained using the network $f($.$) . A pro-$ jection $P(p, v)$ from an arbitrary view point $v$ is obtained by performing a perspective transformation and projecting the transformed point cloud on to a plane. The view-point is parametrized by the camera calibration matrix and extrinsic parameters $K$ and $(R, t)$ respectively. We assume the knowledge of these parameters in the training stage. Let $N$ be the number of points in the point cloud. Then the transformed point $\hat{p}_{n}=\left(\hat{x}_{n}, \hat{y}_{n}, \hat{z}_{n}\right)$ in the camera coordinates is obtained as follows:

$$
\hat{p}_{n}=K\left(R_{v} p_{n}+t_{v}\right) \quad \forall n \in\{1, \cdots, N\}
$$

To train the $3 \mathrm{D}$ reconstruction network, the ground truth $2 \mathrm{D}$ mask, $M$ is used to supervise the projection, $\hat{M}=P(p, v)$.

\section{Continuous Approximation Projection}

The 3D reconstruction network consists of an encoder which takes in a 2D image as input, followed by a decoder which reconstructs the point cloud (Fig. 1). The predicted points are projected from $V$ different view-points and the loss is 


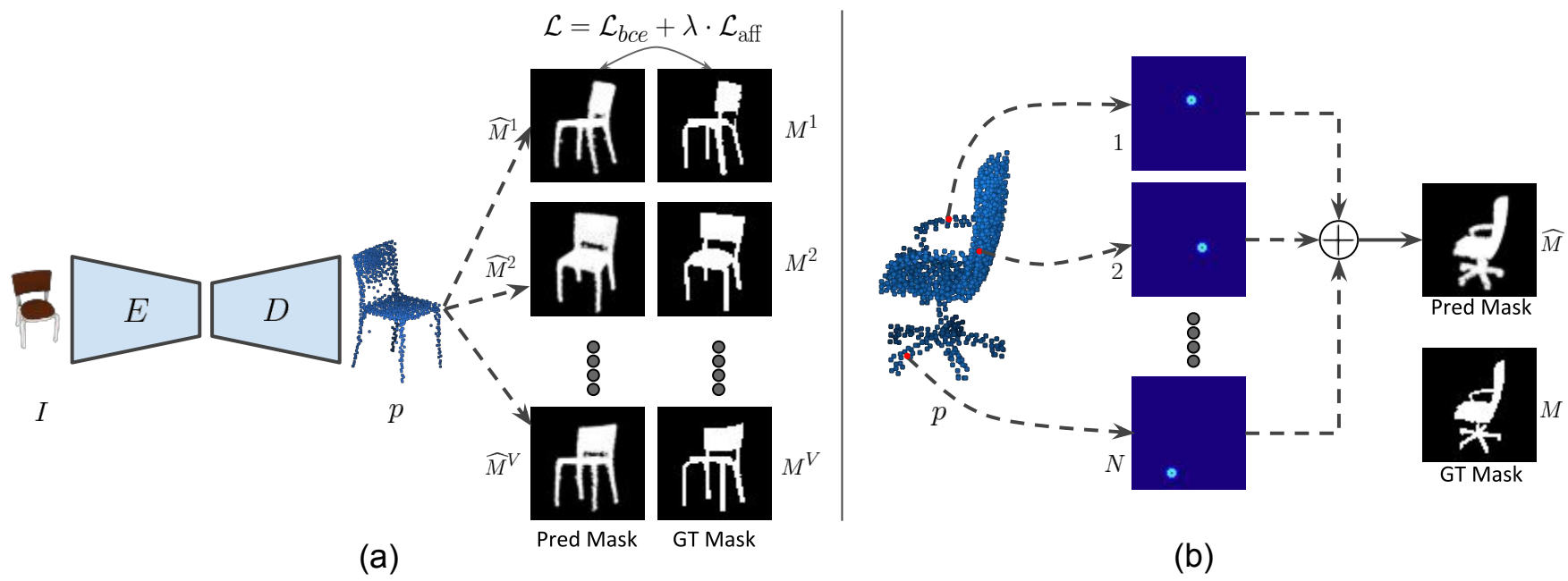

Figure 1: Network architecture and projection module: (a) An encoder-decoder architecture is used to obtain point cloud reconstructions from a $2 \mathrm{D}$ image. The point cloud is projected from multiple view points and compared with corresponding ground truth mask. We use a combination of binary cross-entropy and point affinity loss as our projection consistency loss. (b) An overview of our projection module is shown. Each point in the prediction (red dot in the image) is projected on to a 2D grid by generating a Gaussian map centered at the (x,y) location of the the point. The Gaussian maps from all the points are then combined to obtain a smooth projection that matches the ground truth. Image best viewed zoomed and in color.

calculated with the corresponding ground truth projections. Let $\hat{M}_{i, j}^{v}$ be the pixel value of $v^{t h}$ projected mask at $(i, j)$ coordinates. The projected mask is obtained as follows:

$$
\hat{M}_{i, j}^{v}=\tanh \left(\sum_{n=1}^{N} \phi\left(\hat{x}_{n}-i\right) \phi\left(\hat{y}_{n}-j\right)\right)
$$

where $\tanh$ is the hyperbolic tangent function and $\phi($.$) is a$ kernel function. To obtain a smooth projection, we use an un-normalized Gaussian kernel of variance $\sigma^{2}$ :

$$
\phi(k)=\exp \left(\frac{-k^{2}}{2 \sigma^{2}}\right)
$$

The variance of the kernel is set such that the projection is smooth while retaining the finer structures present in the ground truth mask. Refer to Discussion Section for details.

The proposed point cloud rendering module is significantly different from the existing volumetric approaches for the following reasons: (1) Unlike an ordered voxel representation, a point cloud does not reside in a discretized grid world, but rather in continuous free space. A direct application of volumetric rendering would require embedding the point cloud in a 3D grid, and such a discretization operation is non-differentiable, preventing back-propagation in neural networks. We navigate this problem by treating the value at every pixel to be a continuous approximation of the points in the point cloud. (2) Volumetric rendering in (Yan et al. 2016) is handled by the Spatial Transformer Network (STN) (Jaderberg et al. 2015), which performs bilinear interpolation at the grid cell corners. Apart from being non-differentiable for point sets, this approach would produce 'holes' in the projection for low density point clouds. On the contrary, we introduce a continuous approximation module which utilizes a Gaussian kernel to obtain smooth and accurate projections (Discussion Section, Fig. 7b).

\section{Loss Formulation}

We enforce consistency between the projected and ground truth maps using the binary cross-entropy loss, given by:

$$
\mathcal{L}_{\text {bce }}=\sum_{v=1}^{V}-M^{v} \log \hat{M}^{v}-\left(1-M^{v}\right) \log \left(1-\hat{M}^{v}\right)
$$

where $M^{v}$ and $\hat{M}^{v}$ are the ground truth and predicted masks respectively of dimension $(H, W)$. However, we observe that training the network with just $\mathcal{L}_{b c e}$ results in reconstructions with a large number of outlier points. To alleviate this effect, we propose a loss function that penalizes outlier points in the projected maps by enforcing a nearest point affinity loss, defined as follows:

$$
\begin{aligned}
\mathcal{L}_{\mathrm{aff}} & =\sum_{v=1}^{V} \sum_{i, j}^{H, W} \min _{(k, l) \in M_{+}^{v}}\left((i-k)^{2}+(j-l)^{2}\right) \hat{M}_{i, j}^{v} M_{k, l}^{v} \\
& +\sum_{v=1}^{V} \sum_{i, j}^{H, W} \min _{(k, l) \in \hat{M}_{+}^{v}}\left((i-k)^{2}+(j-l)^{2}\right) M_{i, j}^{v} \hat{M}_{k, l}^{v}
\end{aligned}
$$

where $M_{+}^{v}$ and $\hat{M}_{+}^{v}$ are sets of pixel coordinates of the ground truth and predicted projections whose values are non-zero. Intuitively, this constraint minimizes the nearest neighbour distance between two pixel maps weighted by pixel confidence. We observe that the use of $\mathcal{L}_{\text {aff }}$ is critical in obtaining meaningful reconstructions (Discussion Section). Regions in the mask where ground truth confidence is one, 
but the prediction is near zero, might result in weak gradients if there are no predicted points in the nearby region. Similar issues arise when an incorrect isolated prediction is present. Affinity loss helps in alleviating both these issues. The final loss function during optimization is a combination of binary cross-entropy and affinity loss:

$$
\mathcal{L}=\mathcal{L}_{\text {bce }}+\lambda \cdot \mathcal{L}_{\text {aff }}
$$

\section{Test-Stage Optimization (TSO)}

While the reconstructions obtained by training a network as described above are reasonably correct, they fail to exactly match the input image. Existing state-of-the-art approaches also produce outputs that do not correspond completely, and often fail to reconstruct finer details visible in the image. We propose a test-stage optimization procedure that starts from an initial point cloud $y$, that is the output from any point cloud prediction network, and outputs an optimized point cloud $\hat{y}$. This procedure aims to exactly match the reconstructed point cloud in the region visible in the input image, while obtaining meaningful structure in the occluded regions. To achieve this, we match the projection of the point cloud from the input image view. We make the reasonable assumption of the presence of ground truth mask for the input image in the test stage. We explore three ways of updating the predicted point cloud:

(1) Update only $E$ : Keeping the decoder fixed, we only update the parameters of the encoder by optimizing for the binary cross entropy loss $\mathcal{L}_{\text {bce }}$ (Eqn. 4 ).

(2) Update $E$ and $D$ : We update the parameters of both the encoder and decoder to match the projection. In order to preserve the structure in the occluded regions, we additionally employ a regularization loss on the update of the point cloud. The total loss is defined as

$$
\mathcal{L}_{\text {tso }}=\mathcal{L}_{\text {bce }}+\gamma \cdot d(y, \hat{y})
$$

where $d($.$) is a distance function, y$ and $\hat{y}$ are the initial and optimized point clouds respectively.

(3) Directly update $p$ : We directly update the predicted points using Eqn. 7, without updating any of the network parameters. This approach enables the optimization procedure to be utilized even in cases where the point cloud prediction network is not available during inference.

\section{Experiments}

\section{D Reconstruction on ShapeNet}

Implementation details We fix the number of projections to four in all the experiments. The view-points are randomly selected as in (Tulsiani et al. 2017). The kernel variance $\sigma^{2}$ in Eqn. 3 is chosen as [0.4, 0.4,0.1] for [chair,car,airplane] in the single-category experiments and 0.4 in the multicategory experiments. $\lambda$ is set to 1 in Eqn. 6. We use Adam optimizer to train the network with a learning rate of $5 e^{-5}$. The network architecture details are provided in the supplementary material. For the test-stage optimization procedure, we experiment with different hyperparameter settings for each of the three variants and choose settings that are optimal for each. Learning rates are set to $1 e^{-6}, 5 e^{-6}$, and $5 e^{-4}$ for variants 1,2 and 3 respectively. The weightage for regularization is set to $1 e^{6}$ in Eqn. 7. The optimization procedure is run for 50 iterations and takes $1 \mathrm{~s}$ on an Nvidia GTX 1080Ti GPU.

Dataset We use the textured 3D CAD models from the synthetic ShapeNet dataset (Chang et al. 2015) for our experiments. We consider three exemplar categories: airplane, car and chair. We follow the set-up of (Tulsiani et al. 2017) and use the same train/val/test splits so as to be comparable to existing works.

Evaluation Methodology We use the Chamfer distance between point clouds as our metric to evaluate reconstruction. The Chamfer distance between two point clouds $\widehat{P}$ and $P$ is defined as $d_{\text {Chamfer }}(\widehat{P}, P)=$ $\sum_{x \in \widehat{P}} \min _{y \in P}\|x-y\|_{2}^{2}+\sum_{x \in \widehat{P}} \min _{y \in P}\|x-y\|_{2}^{2}$. The ground truth point cloud is obtained by randomly sampling 16,384 points on the surface of the object and performing farthest point sampling to obtain 1024 points. To evaluate approaches which reconstruct voxelized representations, we use the code provided by (Sun et al. 2018) to convert them to point clouds. The procedure consists of first generating an iso-surface from the voxels using the Marching Cubes algorithm (Lorensen and Cline 1987), and then sampling points on the generated surface to obtain the final point cloud.

Comparison We benchmark our proposed approach against state-of-the-art 3D and 2D supervision works. For 3D supervision, we compare our work with the fullyconnected decoder variant of (Fan, Su, and Guibas 2017), hereby referred to as PSGN-FC. The PSGN-FC network is trained with 3D point cloud supervision, and Chamfer distance is used as the loss function. We follow the same network architecture that is used for our 2D supervision experiments. For evaluating our approach against 2D supervision works, we compare with DRC (Tulsiani et al. 2017), which outputs voxelized $3 \mathrm{D}$ reconstructions. To evaluate DRC, we use the pre-trained models provided by the authors, and convert the representations to the point cloud format as described in the evaluation section. We also compare against Lin et al. (Lin, Kong, and Lucey 2018), who fuse depth maps from eight fixed views to obtain the point cloud. Since Lin et al. predictions are dense, we apply farthest point sampling on the outputs to obtain 1024 points for evaluation.

Results Table 1 presents the results on the ShapeNet dataset with comparisons against the 3D-supervised PSGNFC, 2D-supervised DRC and depth-fusion-based Lin et al. We significantly outperform DRC while achieving results comparable to the 3D supervised PSGN-FC. It is interesting to note that our approach, with just foreground masks as supervision, outperforms even the depth-based approaches of DRC and Lin et al. Fig. 2 shows qualitative results on 
Table 1: Chamfer metrics on ShapeNet (all values are scaled by 1000). We significantly outperform both mask and depth variants of DRC, and obtain scores close to 3D supervised PSGN-FC. In comparison to Lin et al., who fuse depth maps from eight fixed views, we obtain better performance using just mask projection from four random views.

\begin{tabular}{llllll}
\hline Method & Supervision & Airplane & Car & Chair & Mean \\
\hline PSGN-FC (Fan, Su, and Guibas 2017) & 3D & 1.36 & 1.40 & 3.98 & 2.25 \\
PSGN-FC (multi-cat) (Fan, Su, and Guibas 2017) & 3D & 1.33 & 1.41 & 3.94 & 2.23 \\
\hline DRC-Depth (Tulsiani et al. 2017) & Depth & 6.30 & 4.33 & 11.38 & 7.34 \\
Lin et al. (Lin, Kong, and Lucey 2018) & Depth & 2.01 & 2.50 & 6.35 & 3.62 \\
\hline DRC-Mask (Tulsiani et al. 2017) & Mask & 18.94 & 4.92 & 15.91 & 13.26 \\
Ours & Mask & $\mathbf{2 . 0 0}$ & $\mathbf{1 . 6 5}$ & $\mathbf{4 . 4 2}$ & $\mathbf{2 . 6 9}$ \\
Ours (multi-cat) & Mask & 2.57 & 1.74 & 5.09 & 3.13 \\
\hline
\end{tabular}
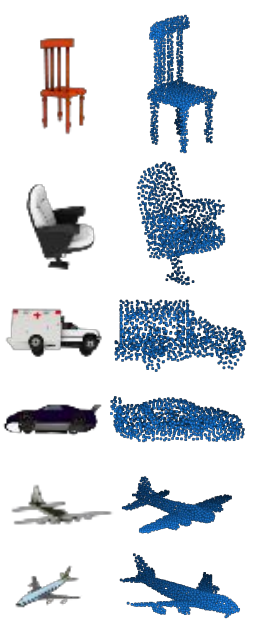

Input

GT
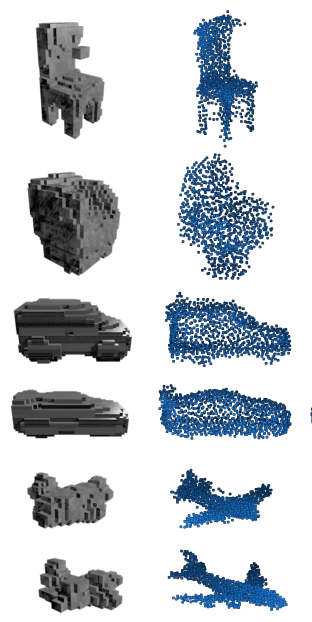

DRC

Lin et al.

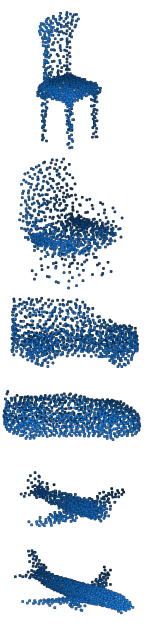

PSGN-FC

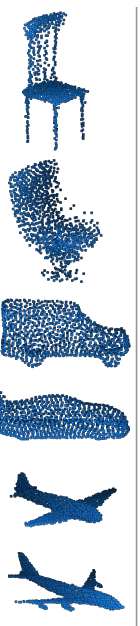

Ours

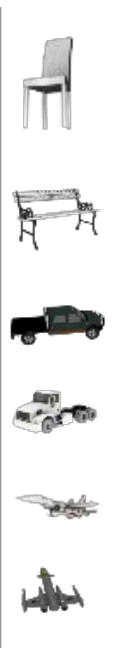

Input
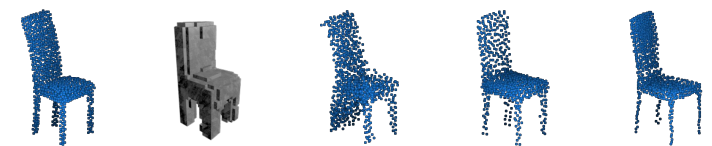

Figure 2: Qualitative comparison on ShapeNet. Our network achieves better reconstructions, with high correspondence to the input image. While DRC outputs are blobby and lack concavity, Lin et al. predictions are rough and have a number of outlier points. In contrast, our predictions are sharper and more accurate. Our network also predicts finer structures like bars on the back of chairs, which the 3D-supervised network fails to capture.

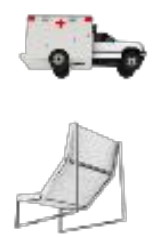

Input

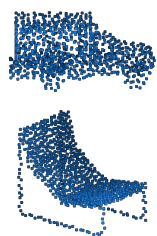

GT

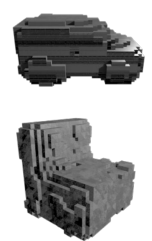

DRC

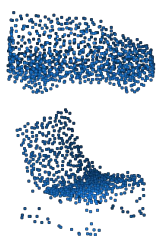

PSGN-FC

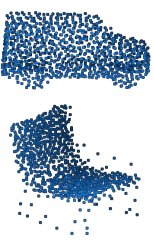

Ours
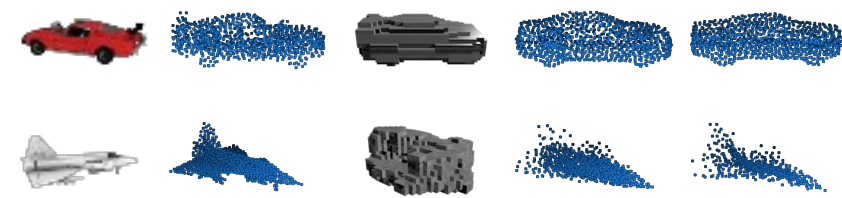

Input

GT

DRC

PSGN-FC

Ours

Figure 3: Qualitative results for the multi-category experiment on ShapeNet.

ShapeNet. We observe that unlike DRC, our approach accurately reconstructs structures with concavities. Lin et al. predictions are rough and have a number of outlier points, while our predictions are sharper and more accurate. Our network is also able to better predict finer details, e.g. hollow regions in the back of chairs, which the 3D-supervised method fails to capture. We also train a single network on all three categories. We observe that the network performs comparably to the single category variants (Table 1 and Fig.3).

\section{D Reconstruction on Pix3D}

To show the efficacy of our approach on real data, we consider Pix3D, a real world dataset with 3D CAD models and corresponding 2D images from multiple view-points. We use the 3D models only for evaluation. We randomly select $80 \%$ of the dataset as the train set and the rest as the test set. 


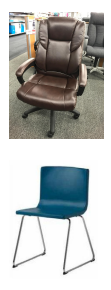

Input

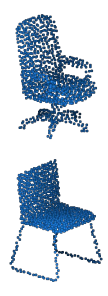

GT

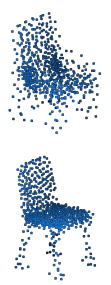

PSGN-FC

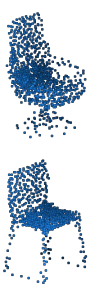

Ours

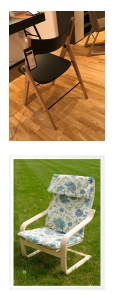

Input

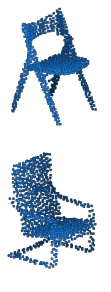

GT

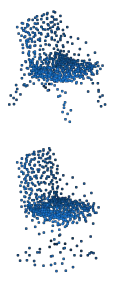

PSGN-FC

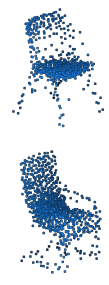

Ours

Figure 4: Qualitative comparison on the real-world Pix3D dataset (Sun et al. 2018). Our network fine-tuned with just a single mask as supervision, is able to effectively reconstruct from real world images. Shapes and finer details are better captured in comparison to the 3D supervised network trained only on ShapeNet.

(a)

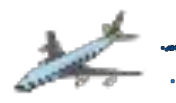

(b)

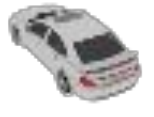

Input
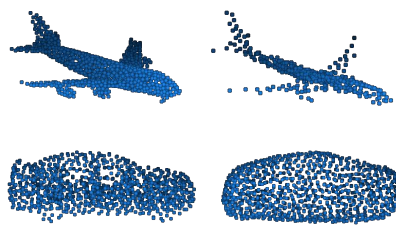

GT

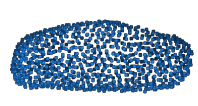

Ours

Figure 5: Failure modes. (a) Multi-category training produces narrower airplanes. (b) Cars trained with just a single mask as supervision have slightly deformed bodies.

Table 2: Chamfer metrics on Pix3D (all values are scaled by 1000). (Fwd: GT $\rightarrow$ Pred, Bwd: Pred $\rightarrow$ GT.

Chamfer: Fwd+Bwd.)

\begin{tabular}{llll}
\hline Method & Fwd & Bwd & Chamfer \\
\hline PSGN-FC & 5.04 & 5.06 & 10.1 \\
Ours(joint) & $\mathbf{4 . 4 4}$ & $\mathbf{4 . 7 6}$ & $\mathbf{9 . 2}$ \\
\hline
\end{tabular}

We show that fine-tuning a PSGN-FC network pre-trained on ShapeNet with the additional 2D training data results in improved performance. To adapt to the domain of real world images, the PSGN-FC network is trained with synthetic ShapeNet images overlaid on random natural backgrounds, as done in (Tulsiani et al. 2017). Results are reported in Table 2. Qualitative results are presented in Fig. 4. We observe that as in the case of the synthetic dataset, our approach results in more faithful reconstructions. The finer details present in the leg and handle regions of chairs are effectively captured. This demonstrates the applicability of

Table 3: Chamfer metrics on test stage optimization (metrics are scaled by 1000). (Fwd: GT $\rightarrow$ Pred, Bwd: Pred $\rightarrow$ GT. Chamfer: Fwd+Bwd.)

\begin{tabular}{llll}
\hline Method & Fwd & Bwd & Chamfer \\
\hline Pre-trained Net & 2.45 & 2.19 & 4.64 \\
TSO - update $E$, fix $D$ & 2.29 & 2.14 & 4.43 \\
TSO - update $E$ and $D$ & $\mathbf{2 . 2 8}$ & $\mathbf{2 . 0 9}$ & $\mathbf{4 . 3 7}$ \\
TSO - directly update $p$ & 2.36 & 2.13 & 4.49 \\
\hline
\end{tabular}

our training methodology for real world scenarios.

\section{Test Stage optimization}

To evaluate our test stage optimization (TSO) approach, we train a PSGN-FC network to reconstruct point clouds aligned with the input image view. We then perform the optimization step at the test stage for every input image. We set the distance function $d$ in Eqn. 7 to be the Chamfer distance between the initial and optimized point clouds in all our experiments. Table 3 shows quantitative metrics for the TSO variant where both the encoder and decoder are updated by optimizing the projection and regularization losses (Eqn. 7). We report the Chamfer distance along with the corresponding forward and backward losses on the test set. We observe that all three variants of TSO lead to improved performance compared to the intial prediction from the pre-trained network. In our qualitative study, we observed that the TSO variant with the decoder fixed had limited capacity to capture the finer details present in the image, while the other two variants $((E, D)$ updated, and only $p$ updated $)$ performed better in generating point clouds that correspond to the input image. Fig. 6 shows qualitative results on samples from the test set before and after running the optimization routine. We observe that the reconstructions match the input image from the visible view, while preserving the structure in the occluded regions. The optimized point clouds display geometric and structural details (curved backrest, bars, etc) present in the input image, that are absent in the initial predictions.

\section{Discussion}

Variance of kernel We plot the category-averaged $\mathcal{L}_{1}$ error between the ground truth mask and corresponding projection for different $\sigma^{2}$ values (Eqn. 3) in Fig. 9. Projections for a sample model are shown in Fig. 7c. Lower $\sigma^{2}$ values result in holes whereas higher values fill large areas. $\sigma^{2}$ is set to 0.4 for chair and car, and 0.1 for airplane. In the multicategory setup, it is set to 0.4 for optimal performance. For comparison, we also display the obtained projection map from a naive discretization-based method (Fig. 7a) and Lin et al.'s method (with upsampling factor $U=5$ ) (Fig. 7b).

Effect of number of projections We study the effect of number of ground truth projections per image used during training (Table 4). We observe that the network is able to 


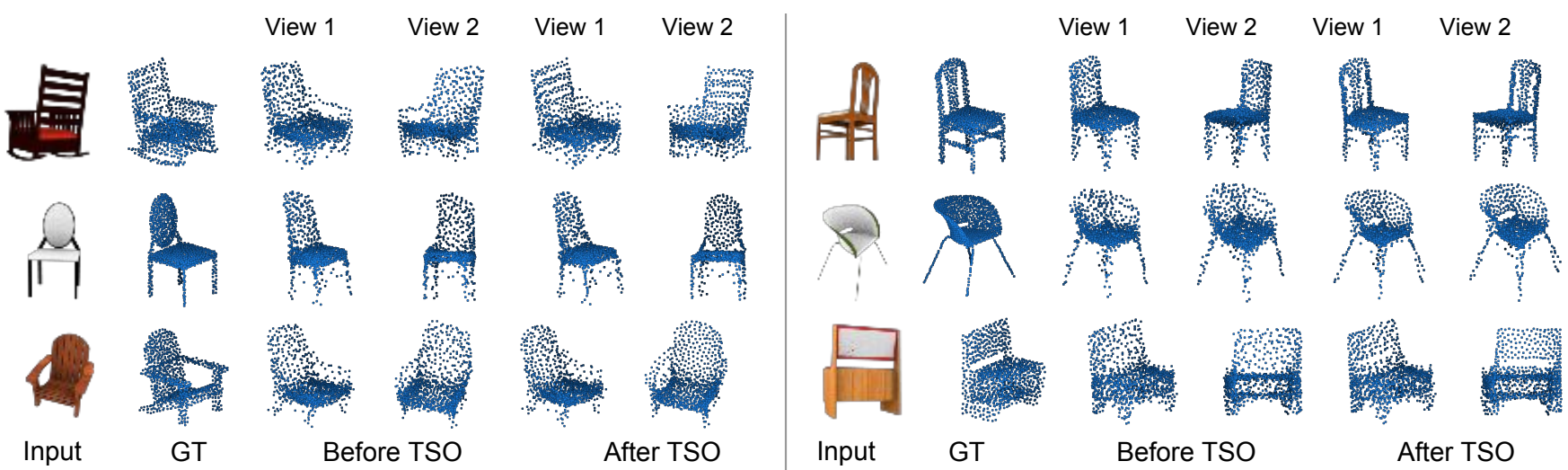

Figure 6: Qualitative results showing the generated point clouds before and after test-stage optimization (TSO). The optimized point clouds display geometric and structural details (curved backrest, bars, etc) present in the input image, that are absent in the initial predictions. Reconstruction results are shown from two different viewing angles so as to highlight the retention of structure when seen from a different view and the correspondence with the input image when seen from the same view.

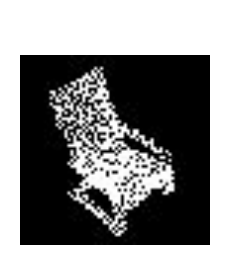

(a) Discretization

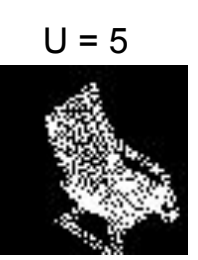

(b) Lin et al.

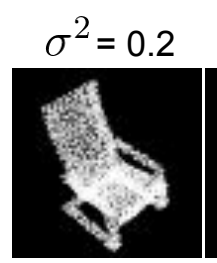

(c) Ours at different $\sigma^{2}$ values

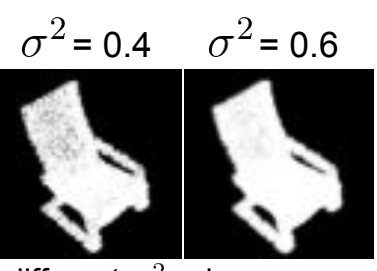

Ground truth

Figure 7: Projection maps for discretized projection, Lin et al., and our method. (a) and (b) have a lot of 'holes'. (c) Our projections are smooth due to the continuous approximation module. Here, $\sigma^{2}=0.4$ fills in holes while retaining finer details.

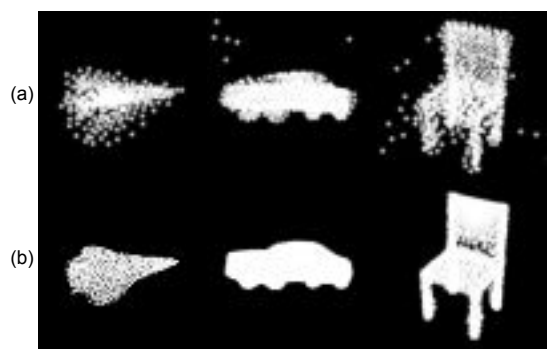

Figure 8: Projected predictions trained (a) without and (b) with affinity loss, which helps in outlier point removal.

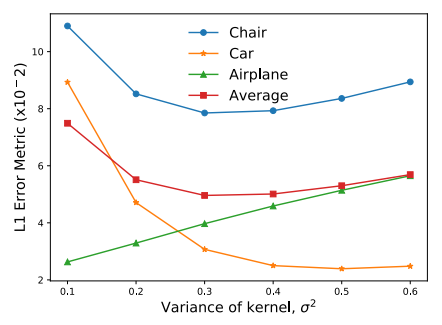

Figure 9: Variance of kernel, $\sigma^{2}$ vs $\mathcal{L}_{1}$ error for mask projections plotted for different categories.
Table 4: Dependency of reconstruction performance on number of views. Chamfer scores are scaled by 1000

\begin{tabular}{llllllll}
\hline Num Views & 1 & 2 & 3 & 4 & 5 & 6 & 8 \\
\hline Airplane & 2.40 & 2.02 & 2.0 & 2.0 & 1.98 & 1.99 & 2.01 \\
Car & 3.47 & 1.68 & 1.65 & 1.65 & 1.68 & 1.65 & 1.68 \\
Chair & 4.53 & 4.41 & 4.35 & 4.36 & 4.43 & 4.42 & 4.56 \\
\hline
\end{tabular}

reconstruct well with just a single mask as supervision. The performance improves with two masks and stays constant with higher number of projections.

Role of Affinity Loss To analyze the role of the affinity loss $\mathcal{L}_{\text {aff }}$ (Eqn. 5), we train a network with only cross entropy loss $\mathcal{L}_{\text {bce }}$ (Eqn. 4). We observe that the reconstructions are noisy with a lot of outlier points, resulting in higher Chamfer scores. The addition of $\mathcal{L}_{\text {aff }}$, results in better reconstructions (Fig. 8). However, in the case of chairs, we notice that a high weightage to $\mathcal{L}_{\text {aff }}$ delays the emergence of thinner structures like legs. Hence, we reduce the the weight of $\mathcal{L}_{\text {aff }}$ after a fixed number of iterations. The loss weighing strategy between $\mathcal{L}_{\text {bce }}$ and $\mathcal{L}_{\text {aff }}$ is provided in the supplementary.

Failure modes We analyze the failure modes for our method in Fig. 5. (a) Certain instances of airplanes have a 
narrower body in comparison to the ground truth for multi-category trained models. Choosing $\sigma^{2}$ values per category alleviates this effect. (b) Cars trained with only single view mask supervision $(\mathrm{V}=1)$ have a slight deformation in the depth dimension. Using an additional view during supervision corrects this.

\section{Conclusion}

We introduced a continuous approximation projection module for a differentiable and accurate rendering of 3D point clouds, to enable weakly supervised 3D object reconstruction. A loss formulation was introduced in the training procedure to improve reconstructions. We highlighted the utility of such an approach in real world scenarios by improving the reconstruction performance using as little as a single mask as supervision. Quantitative and qualitative evaluation on synthetic and real-world datasets show that the generated point clouds are of better quality in comparison to the current state-of-art projection-based reconstruction methods. Furthermore, we also demonstrated that the presence of object masks at test stage can be utilized to obtain highly corresponding 3D reconstructions. In the future, we would like to explore ways of extending the projection framework to obtain depth maps, color images and any other features associated with the object.

\section{Acknowledgement}

This work was funded by the Robert Bosch Centre for Cyber Physical Systems, IISc (Project ID: RBCO0052).

\section{References}

Chang, A. X.; Funkhouser, T.; Guibas, L.; Hanrahan, P.; Huang, Q.; Li, Z.; Savarese, S.; Savva, M.; Song, S.; Su, H.; et al. 2015. Shapenet: An information-rich 3D model repository. arXiv preprint arXiv:1512.03012.

Choy, C. B.; Xu, D.; Gwak, J.; Chen, K.; and Savarese, S. 2016. 3D-r2n2: A unified approach for single and multi-view 3D object reconstruction. In European Conference on Computer Vision, 628 644. Springer.

Fan, H.; Su, H.; and Guibas, L. 2017. A point set generation network for 3D object reconstruction from a single image. In Conference on Computer Vision and Pattern Recognition (CVPR), volume 38.

Girdhar, R.; Fouhey, D. F.; Rodriguez, M.; and Gupta, A. 2016. Learning a predictable and generative vector representation for objects. In European Conference on Computer Vision, 484-499. Springer.

Girshick, R.; Donahue, J.; Darrell, T.; and Malik, J. 2014. Rich feature hierarchies for accurate object detection and semantic segmentation. In Conference on Computer Vision and Pattern Recognition (CVPR).

Goodfellow, I.; Pouget-Abadie, J.; Mirza, M.; Xu, B.; WardeFarley, D.; Ozair, S.; Courville, A.; and Bengio, Y. 2014. Generative adversarial nets. In Advances in neural information processing systems.

Groueix, T.; Fisher, M.; Kim, V. G.; Russell, B.; and Aubry, M. 2018. AtlasNet: A Papier-Mâché Approach to Learning 3D Surface Generation. In CVPR.
Häne, C.; Tulsiani, S.; and Malik, J. 2017. Hierarchical surface prediction for 3D object reconstruction. arXiv preprint arXiv:1704.00710.

Jaderberg, M.; Simonyan, K.; Zisserman, A.; et al. 2015. Spatial transformer networks. In Advances in neural information processing systems.

Kato, H.; Ushiku, Y.; and Harada, T. 2017. Neural 3D mesh renderer. arXiv preprint arXiv:1711.07566.

Krizhevsky, A.; Sutskever, I.; and Hinton, G. E. 2012. Imagenet classification with deep convolutional neural networks. In $A d$ vances in neural information processing systems, 1097-1105.

Li, Y.; Bu, R.; Sun, M.; and Chen, B. 2018. PointCNN. arXiv preprint arXiv: 1801.07791.

Lin, C.-H.; Kong, C.; and Lucey, S. 2018. Learning efficient point cloud generation for dense $3 \mathrm{D}$ object reconstruction. In AAAI Conference on Artificial Intelligence (AAAI).

Long, J.; Shelhamer, E.; and Darrell, T. 2015. Fully convolutional networks for semantic segmentation. In Proceedings of the IEEE conference on computer vision and pattern recognition.

Lorensen, W. E., and Cline, H. E. 1987. Marching cubes: A high resolution $3 \mathrm{~d}$ surface construction algorithm. In ACM siggraph computer graphics, volume 21, 163-169. ACM.

Mandikal, P., and Babu, R. V. 2019. Dense 3d point cloud reconstruction using a deep pyramid network. In Winter Conference on Applications of Computer Vision (WACV).

Mandikal, P.; K L, N.; Agarwal, M.; and Babu, R. V. 2018. 3DLMNet: Latent embedding matching for accurate and diverse $3 \mathrm{~d}$ point cloud reconstruction from a single image. In Proceedings of the British Machine Vision Conference (BMVC).

Mandikal, P.; K L, N.; and Babu, R. V. 2018. 3D-PSRNet: Part segmented $3 \mathrm{~d}$ point cloud reconstruction from a single image. In $3 D$ Reconstruction Meets Semantics Workshop (ECCVW).

Qi, C. R.; Su, H.; Mo, K.; and Guibas, L. J. 2017a. Pointnet: Deep learning on point sets for 3D classification and segmentation. Proc. Computer Vision and Pattern Recognition (CVPR), IEEE 1(2):4.

Qi, C. R.; Yi, L.; Su, H.; and Guibas, L. J. 2017b. Pointnet++: Deep hierarchical feature learning on point sets in a metric space. In Advances in Neural Information Processing Systems, 5105-5114.

Riegler, G.; Ulusoy, A. O.; and Geiger, A. 2017. Octnet: Learning deep 3D representations at high resolutions. In $C V P R$

Su, H.; Jampani, V.; Sun, D.; Maji, S.; Kalogerakis, V.; Yang, M.H.; and Kautz, J. 2018. Splatnet: Sparse lattice networks for point cloud processing. arXiv preprint arXiv:1802.08275.

Sun, X.; Wu, J.; Zhang, X.; Zhang, Z.; Zhang, C.; Xue, T.; Tenenbaum, J. B.; and Freeman, W. T. 2018. Pix3d: Dataset and methods for single-image $3 \mathrm{~d}$ shape modeling. In CVPR.

Tatarchenko, M.; Dosovitskiy, A.; and Brox, T. 2017. Octree generating networks: Efficient convolutional architectures for highresolution $3 \mathrm{D}$ outputs. In $C V P R$.

Tulsiani, S.; Zhou, T.; Efros, A. A.; and Malik, J. 2017. Multiview supervision for single-view reconstruction via differentiable ray consistency. In $C V P R$.

Wu, J.; Wang, Y.; Xue, T.; Sun, X.; Freeman, B.; and Tenenbaum, J. 2017. Marrnet: 3D shape reconstruction via $2.5 \mathrm{~d}$ sketches. In Advances In Neural Information Processing Systems, 540-550.

Yan, X.; Yang, J.; Yumer, E.; Guo, Y.; and Lee, H. 2016. Perspective transformer nets: Learning single-view 3D object reconstruction without 3D supervision. In Advances in Neural Information Processing Systems. 